\title{
Hierarchical formation of Dark Matter Halos near the Free Streaming Scale, and Their Implications on Indirect Dark Matter Search
}

\author{
Tomoaki Ishiyama \\ Center for Computational Science, University of Tsukuba, 1-1-1, Tennodai, Tsukuba, Ibaraki, \\ 305-8577, Japan \\ email: ishiyama@ccs.tsukuba.ac.jp
}

\begin{abstract}
The smallest dark matter halos are formed first in the early universe. According to recent studies, the central density cusp is much steeper in these halos than in larger halos and scales as $\rho \propto r^{-(1.5-1.3)}$. We present results of very large cosmological $N$-body simulations of the hierarchical formation and evolution of halos over a wide mass range, beginning from the formation of the smallest halos. We confirmed early studies that the inner density cusps are steeper in halos at the free streaming scale. The cusp slope gradually becomes shallower as the halo mass increases. The slope of halos 50 times more massive than the smallest halo is approximately -1.3 . The concentration parameter is nearly independent of halo mass, and ruling out simple power law mass-concentration relations. The steeper inner cusps of halos near the free streaming scale enhance the annihilation luminosity of a Milky Way sized halo between 12 to $67 \%$.
\end{abstract}

Keywords. dark matter, halo, numerical

\section{Introduction}

The size of the smallest halo is determined by the free streaming scale of the dark matter particles. If dark matter comprises the lightest supersymmetric particle (the neutralino of mass approximately $100 \mathrm{GeV}$ ), the estimated corresponding mass of the smallest microhalos is approximately earth-mass (e.g. Hofmann et al. 2001; Berezinsky et al. 2003).

If many earth-mass microhalos exist at present universe, they could significantly enhance gamma-ray signals by neutralino self-annihilation. Since the gamma-ray flux is proportional to the square of the local dark matter density, the annihilation signal from the Milky Way halo should largely depend on the halo's fine structure. According to recent studies, the central density cusp is much steeper in the smallest halos than in larger halos (Ishiyama et al. 2010). Such steep cusps may largely impact on indirect experimental searches for dark matter.

In Ishiyama et al. (2010), only three microhalos were simulated. Statistical study, such as the distribution of microhalo density profiles, requires a more extensive dataset. Ishiyama et al.(2010) analytically estimated that the formation epoch of microhalos is affected by larger scale density fluctuations. They predicted that the formation epoch of their simulated microhalos was later than the average value. Since halo concentration reflects the cosmic density at which halos collapse, this suggests that their microhalos were less concentrated than indicated by the average. Therefore, to precisely predict the gamma-ray flux, the distribution of both the microhalo density profile shapes and of microhalo concentrations must be elucidated. 


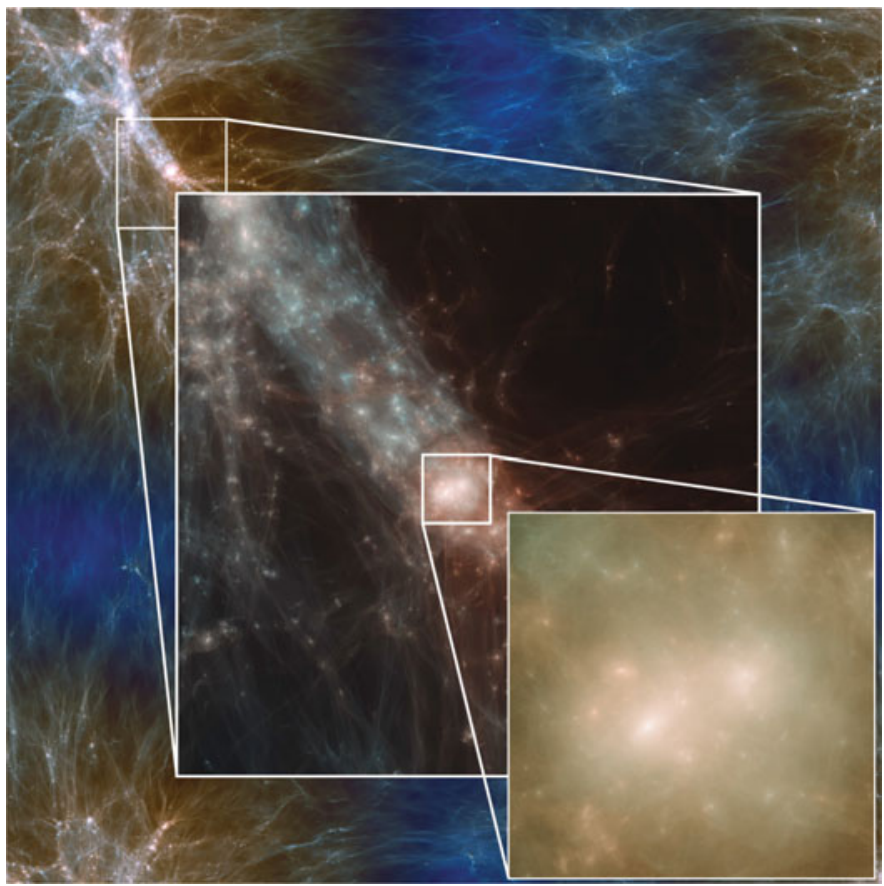

Figure 1. The distribution of dark matter in the A_N4096L400 simulation at $z=32$. Central and bottom right panels show the enlargement of the largest halo.

We address these questions by large and high resolution cosmological $N$-body simulations. The detailed explanation of this work can be found in Ishiyama (2014).

\section{Numerical Methods}

We performed three large cosmological $N$-body simulations by massively parallel TreePM code, GreeM (Ishiyama et al. 2012). Two different initial matter power spectra were used. In two of the simulations, the power spectrum included the sharp cutoff imposed by the free streaming damping of dark matter particles with a mass of $100 \mathrm{GeV}$ (Green et al. 2004). The third simulation ignored the effect of free streaming damping. The initial conditions were generated by a first-order Zeldovich approximation at $z=400$.

In the simulations with the cutoff imposed, the motions of $4096^{3}$ particles in comoving boxes of side lengths $400 \mathrm{pc}$ and $200 \mathrm{pc}$ were followed (these simulations are denoted A_N4096L400 and A_N4096L200, respectively). The particle masses were $3.4 \times 10^{-11} M_{\odot}$ and $4.3 \times 10^{-12} M_{\odot}$, ensuring that halos at the free streaming scale were represented by $\sim 30,000$ and $\sim 230,000$ particles, respectively. The simulation with no cutoff followed the motions of $2048^{3}$ particles in comoving boxes of side length $200 \mathrm{pc}$ (this simulation is denoted B_N2048L200). Figure 1 shows the dark matter distribution in the A_N4096L400 simulation at $z=32$.

\section{Results}

We calculated the spherically averaged radial density profile of each halo within the range $0.02 \leqslant r / r_{\text {vir }} \leqslant 1.0$, divided into 32 logarithmically equal intervals. Each density profile deviates to varying extent from the average density profile, mainly because subhalos exist in the halos. To minimize this effect and obtain proper average radial density 

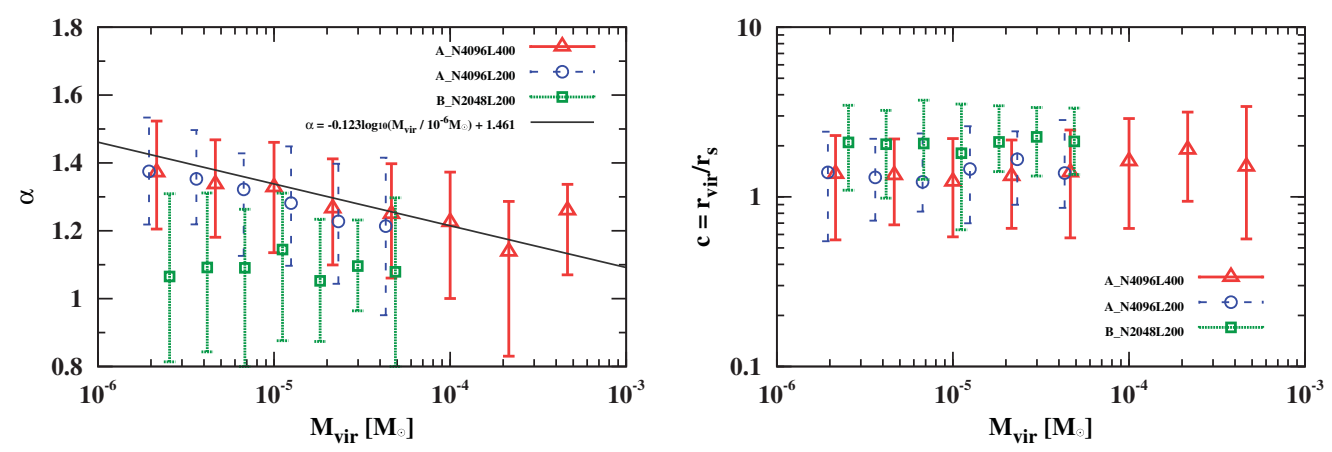

Figure 2. Distributions of slope $\alpha$ and concentration $c=r_{\mathrm{vir}} / r_{\mathrm{s}}$ of the density profile, plotted against the halo virial mass $M_{\mathrm{vir}}$. Circles, triangles and squares show the median value in each mass bin. Whiskers are the first and third quantiles. Black solid line is the best fit power law function [Equation (3.2) in the text].

profiles of halos with a wide mass distribution, we stacked the profiles of similar-mass halos.

To quantify the simulated density structures, we fitted the stacked density profiles to a double power law function, given by

$$
\rho(r)=\frac{\rho_{0}}{\left(r / r_{\mathrm{s}}\right)^{\alpha}\left(1+r / r_{\mathrm{s}}\right)^{(3-\alpha)}} .
$$

The power law functions that best fits the relation between mass and $\alpha$ obtained from our simulations is

$$
\alpha=-0.123 \log \left(M_{\mathrm{vir}} / 10^{-6} M_{\odot}\right)+1.461 .
$$

To visualize the scatter in the density profiles, we fitted the profile of each halo to Equation (3.1) and calculated the median and scatter in each mass bin. These results are shown in Figure 2. The two simulations with different resolutions give similar results (A_N4096L400and A_N4096L200). The median accurately matches the fitting function derived from the stacked density profile. Regardless of halo mass, the first and third quantiles deviate by less than $20 \%$ in the A_N4096L400 simulation. Clearly, the B_N2048L200 simulation generates more scatter than the A_N4096L400 simulation.

Note that the concentration parameter is defined differently than the NFW profile, since another fitting function is used. Remarkably, the concentration parameter in both models is nearly independent of halo mass over the range shown in Figure 2. The median concentration in the cutoff model is $1.2-1.7$, increasing to $1.8-2.3$ without the cutoff.

These results differ from what we see in larger halos (dwarf-galaxy-sized to clustersized halos) at lower redshifts (typically less than $z=5$ ). The concentrations of these halos weakly depend on the halo mass, and have been fitted to many simple single power law functions. Our results rule out single power law mass-concentration relations.

\section{Discussions}

The gamma-ray luminosity of a halo by neutralino self-annihilation seen from a distant observer is calculated by the volume integral of the density squared. The steeper cusps obtained in our simulations could significantly enhance the signals. In this section, we evaluate boost factors using the density profiles obtained in our simulations.

Figure 3 shows the annihilation boost factor as a function of the halo mass. We considered three models. In the first model, we used the profile of Equation (3.1) with the 


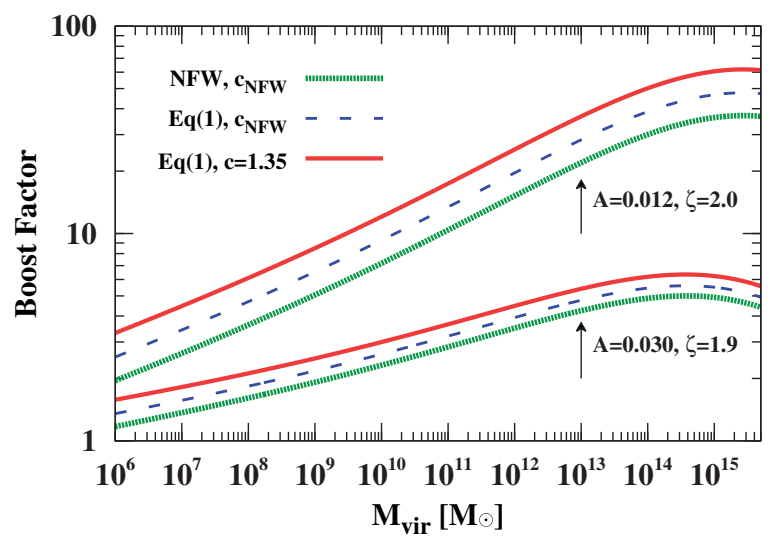

Figure 3. Annihilation boost factor as a function of the halo mass. The results of three models of halos near the free streaming scale are shown. The subhalo mass function $d n / d m=0.012 / M(m / M)^{-2.0}$ gives upper three curves. The subhalo mass function $d n / d m=0.030 / M(m / M)^{-1.9}$ gives lower three curves.

mass-shape relation of Equation (3.2), and the constant concentration $c=1.35$ at $z=32$, consistent with our cutoff simulation. In the second model, we used the profile of Equation (3.1) with the mass-shape relation of Equation (3.2), and the mass-concentration relation proposed by Sánchez-Conde and Prada (2014). In the third model, we used the NFW profile and the mass-concentration relation proposed by Sánchez-Conde and Prada (2014). The models with steeper inner cusps raise the boost factor moderately. The boost factors of a Milky Way sized halo $\left(M=2.0 \times 10^{12} M_{\odot}\right)$ with the $\zeta=2.0$ subhalo mass function are $\sim 17,22$ and 29 for three models, respectively. Those with the $\zeta=1.9$ subhalo mass function are $\sim 3.7,4.2$ and 4.8. Strongly depending on the subhalo mass function and the adopted concentration model, the steeper inner cusps of halos near the free streaming scale enhance the annihilation luminosity of a Milky Way sized halo between 12 to $67 \%$.

\section{Acknowledgement}

Numerical computations were partially carried out on Aterui supercomputer at Center for Computational Astrophysics, CfCA, of National Astronomical Observatory of Japan, and the K computer at the RIKEN Advanced Institute for Computational Science (Proposal numbers hp120286 and hp130026). This work has been funded by MEXT HPCI STRATEGIC PROGRAM and MEXT/JSPS KAKENHI Grant Number 24740115.

\section{References}

Hofmann, S., Schwarz, D. J., \& Stöcker, H. 2001, PRD, 64, 083507

Berezinsky, V., Dokuchaev, V., \& Eroshenko, Y. 2003, PRD, 68, 103003

Ishiyama, T., Makino, J., \& Ebisuzaki, T. 2010, ApJL, 723, L195

Ishiyama, T. 2014, ApJ, 788, 27

Green, A. M., Hofmann, S., \& Schwarz, D. J. 2004, MNRAS, 353, L23

Ishiyama, T., Nitadori, K., \& Makino, J. 2012, in Proc. Int. Conf. High Performance Computing, Networking, Storage and Analysis, SC 12 (Los Alamitos, CA: IEEE Computer Society Press), 5:, (arXiv:1211.4406)

Sánchez-Conde, M. A. \& Prada, F. 2014, MNRAS, 442, 2271 